\title{
Mapping Dispersion Fluctuations along Optical Fibers Using Brillouin Probing and a Fast Analytic Calculation
}

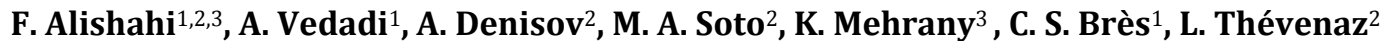 \\ 1. Photonic Systems Laboratory, Ecole Polytechnique Fédérale de Lausanne, CH-1015, Lausanne, Switzerland \\ 2. Group for Fiber Optics, Ecole Polytechnique Fédérale de Lausanne, CH-1015, Lausanne, Switzerland \\ 3. Electronics and Electrical Dept., Sharif University of Technology., Azadi Ave., Tehran, Iran \\ alishahi.fateme@gmail.com
}

\begin{abstract}
A simple analytic formula is derived to extract tiny dispersion fluctuations along highly nonlinear fibers from distributed measurements of parametric gain. A refined BOTDA scheme, suitable to track Kerr processes, enables low noise measurements.

OCIS codes: (060.2270) Fiber optics and optical communications, Fiber characterization; (190.4370) Nonlinear optics, Fibers; (190.4970) Nonlinear optics, parametric oscillators and amplifiers
\end{abstract}

\section{Introduction}

All-optical devices based on nonlinear effects in Highly Nonlinear Fibers (HNLF) are proving to be essential for signal processing at unprecedented speeds and for future all-optical networks. However, it is also known that the performance of such devices can be seriously impeded by tiny longitudinal fluctuations of the zero dispersion wavelength (ZDW). Mapping the ZDW fluctuations along the HNLF is therefore highly desirable for the design of efficient nonlinear effects based devices. Various schemes have been suggested to derive ZDW fluctuations along fibers, mainly using complex distributed sensing of localized nonlinear interactions [1]. In this paper, we propose to utilize the high sensitivity of fiber optical parametric amplifier (FOPA) gain to local dispersion in order to derive the corresponding ZDW fluctuations. In [2] a technique based on Brillouin Optical Time Domain Analysis (BOTDA) was used to probe FOPA gain along the fiber. By improving the experimental scheme, we have been able to measure the FOPA gain along the tested fibers with low noise and high stability. Furthermore, an analytically solvable firstorder differential equation is also provided to extract the ZDW fluctuations. Not only is the dispersion retrieval procedure much faster, it also shows great performance compared to full numerical approaches based on slow curve fitting methods. The combination of the novel setup and formula allows for the extraction of ZDW fluctuations of less than $0.02 \mathrm{~nm}$ with 2 meters resolution.

\section{Experimental setup}

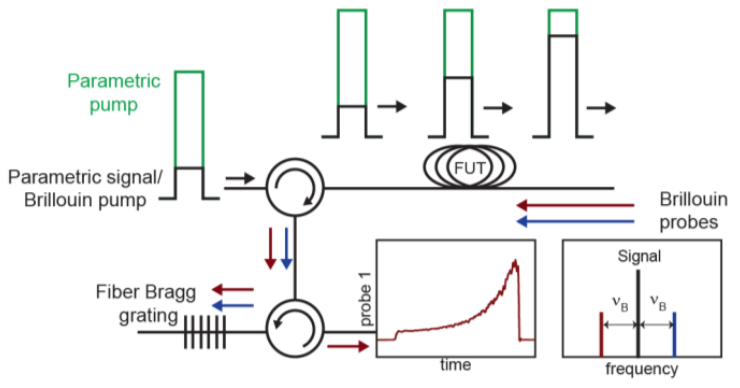

Fig. 1: Experimental setup. FUT: fiber under test
The schematic of the setup is depicted in Fig. 1. Compared to [2], both the FOPA pump and signal are pulsed before being injected into the fiber under test, in our case HNLF. The FOPA signal also acts as a BOTDA pump. Using an electro-optic modulator, two carriersuppressed sidebands are symmetrically generated below and above the BOTDA pump (FOPA signal) frequency. The detuning between the sidebands and the BOTDA pump frequency is set at the Brillouin frequency shift $v_{B}$ of the fiber. The two sidebands, designated as $\mathrm{cw}$ Brillouin probes 1 and 2, are launched at the opposite ends of the fiber. The Brillouin interaction between probes and BOTDA pump leads to a gain on the lower sideband and an identical loss on the higher sideband. As the BOTDA pump power grows along the fiber due to parametric gain, the parametric gain fluctuations along the fiber are imprinted on the Brillouin probes. It should be noted that due to the simultaneous presence of the two probes experiencing opposite gain and loss, the saturation of the BOTDA pump is avoided [3]. A tunable $0.1 \mathrm{~nm}$ bandwidth fiber Bragg grating filter enables the selection of either Brillouin probe. The gain or loss probe trace is finally collected on an oscilloscope for processing. To average out the polarization dependent Brillouin interaction, a polarization switch is used before launching the Brillouin probes into the HNLF. Traces of the gain/loss probe are acquired for the parametric pump switched on and off in order to derive the distributed FOPA gain. Measurements from both sides of the fiber are performed for comparison.

\section{ZDW fluctuations extraction}

In a FOPA, amplification is based on the transfer of energy through four-wave mixing (FWM) from the pump to the signal and a generated idler. The efficiency of the energy transfer depends on the phase matching $\bar{\kappa}$ between the 
interacting waves. Supposing that no pump depletion occurs $\bar{\kappa}=2 \gamma P+\overline{\beta_{2}} \Delta \omega^{2}$, Where $\gamma$ is the fiber nonlinearity coefficient, $P$ is the pump peak power, $\overline{\beta_{2}}$ is the HNLF dispersion and $\Delta \omega$ is the angular frequency detuning between the pump and the signal. $\overline{\beta_{2}}$ fluctuations induce fluctuations on $\bar{\kappa}$ and hence on the FOPA gain. Using a WKB [4] approach, an analytic expression for the FOPA gain in the presence of $\overline{\beta_{2}}$ fluctuations can be obtained. Expanding the FOPA gain to the first Taylor order with respect to the phase matching variations $\Delta \kappa(z)$ and differentiating the whole expression, the following first-order differential equation governing $\Delta \kappa(z)$ is obtained:

$$
d \Delta \kappa / d z-G_{0}^{\prime \prime} / G_{0}^{\prime} \Delta \kappa=4 \bar{g}^{2} / \bar{\kappa} \times 1 / G_{0} \times\left(d \Delta G / d z-G_{0}^{\prime \prime} / G_{0}^{\prime} \Delta G\right)+\left(G_{0}^{\prime \prime} / G_{0}^{\prime}-G_{0}^{\prime} / G_{0}\right) \Delta \kappa(0)
$$

where $\bar{g}$, defined as $\bar{g}^{2}=\gamma P^{2}-\bar{\kappa}^{2} / 4$, is the linear parametric gain, $G_{0}+1=1+(\gamma P / \bar{g} \sinh (\bar{g} z))^{2}$ is the ideal FOPA gain of a fiber with no ZDW fluctuations, $G_{0}^{\prime}$ and $G_{0}^{\prime \prime}$ are the first and second FOPA gain derivatives with respect to the longitudinal position $z$, and $\Delta G$ is the difference between the actual and the ideal FOPA gain. Eq. (1) can be solved analytically, so that:

$$
\Delta \kappa(z)=\mathrm{f}(\Delta G(\mathrm{z}))+\left[\Delta \kappa(0) G_{0}^{\prime}(L-z)+\Delta \kappa(L) G_{0}^{\prime}(\mathrm{z})\right] / G_{0}^{\prime}(L)
$$

where $f(\Delta G(z))=4 \bar{g}^{2} / \bar{\kappa} \times\left[\Delta G(z) / G_{0}(z)-\Delta G(L) / G_{0}(L) \times G_{0}^{\prime}(z) / G_{0}^{\prime}(L)+G_{0}^{\prime}(z) \int_{L}^{z} \Delta G(\xi) / G_{0}(\xi)^{2} d \xi\right]$.

Two different types of HNLFs with lengths $350 \mathrm{~m}$ and $500 \mathrm{~m}$ were tested. Fig. 2(a) \& (c) show the FOPA gain measured in both directions of the $350 \mathrm{~m}$ and $500 \mathrm{~m}$ HNLF, respectively. We observe discrepancies from the ideal FOPA gain that are due to ZDW fluctuations. The insets of Fig. 2(a) show how local FOPA gain variations are related to ZDW fluctuations. Using Eq. (2), the ZDW fluctuations were extracted as shown in Fig. 2(b) \& (d), with a $0.02 \mathrm{~nm}$ accuracy and 2 meters resolution. Except over the $50 \mathrm{~m}$ ends of the tested fibers, the ZDW fluctuations retrieved from both sides of the fibers show coincidence of more than $94 \%$. The erroneous behavior observed at the beginning of the HNLFs is because the idler is yet to be generated and no information on dispersion is available. This problem is overcome by using measurements from both sides of the fiber. Overall, the ZDW fluctuations of both fibers are less than $0.2 \mathrm{~nm}$, in accordance with HNLFs specifications. The $500 \mathrm{~m}$ HNLF exhibits more ZDW fluctuations, which cause stronger FOPA gain variations from the ideal case. We also verified that theoretical gains calculated from the extracted ZDW maps are close to the measured gains with an error of less than $0.2 \%$.
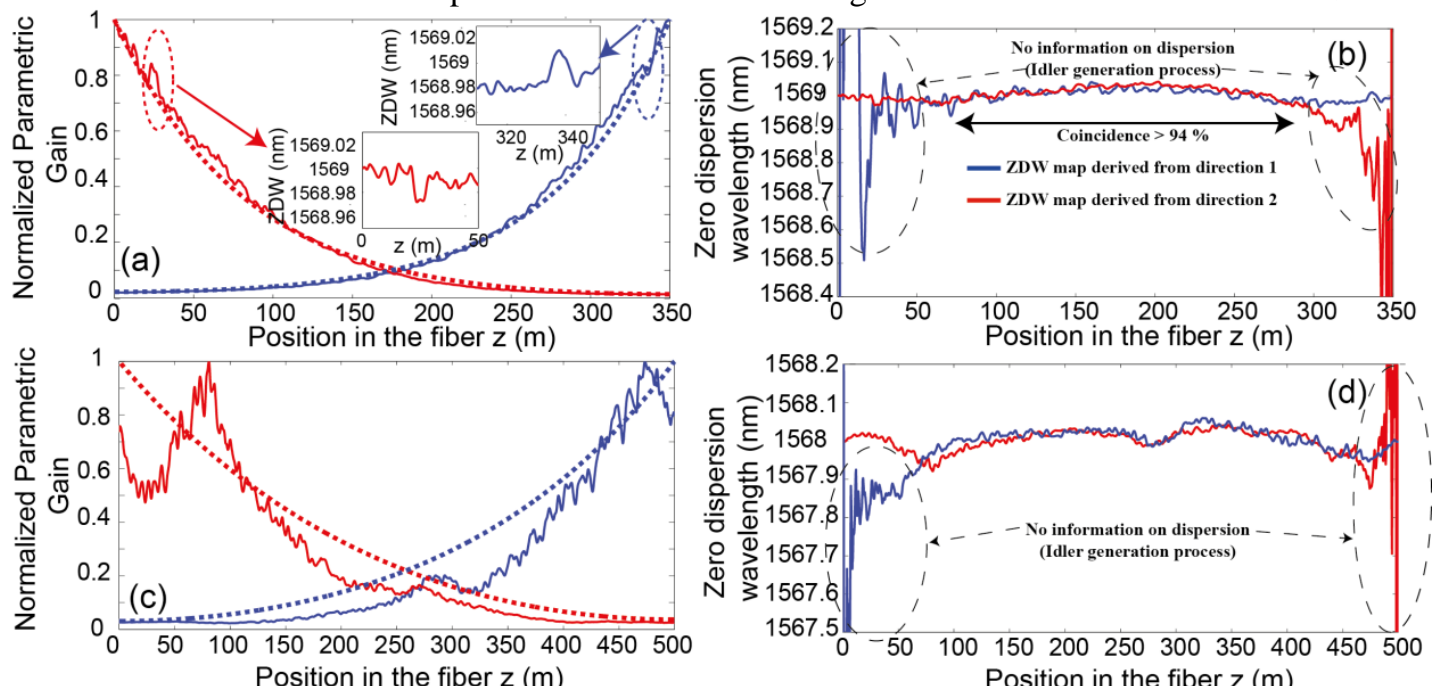

Fig.2 (a,c)Measured FOPA gains (bold lines) FOPA gain of ideal fiber (dotted line)(b,d) dispersion fluctuation for two HNLFs.

\section{Conclusion}

Using a novel scheme based on BOTDA, the distribution of the FOPA gain along HNLFs was measured with better resolution and sensitivity than previously reported. Using an analytical formula, ZDW fluctuation of less than $0.02 \mathrm{~nm}$ could be extracted with a longitudinal resolution of 2 meters. This scheme could also be used to study Kerrbased phenomenon such as FOPA saturation or supercontinuum generation.

The authors acknowledge Sumitomo for providing the 350-m HNLF

\section{Reference}

[1] E. Myslivets, N. Alic, and S. Radic, J. Lightwave Technol., 28, 3478 (2010)

[2] F. Alishahi, A. Vedadi, A. Shoaie, K. Mehrany, J. A. Salehi, Optical Fiber Communication conference, J. Opt. Soc. Am., 2012

[3] K.Song, M. G. Herráez, and L. Thévenaz, J. Lightwave Technol., 23, 4140 (2005)

[4] M. E. Marhic, Fiber optical parametric amplifiers, oscillators and related devices,.(Cambridge University Press, New York, 2008) 